\title{
Study on the Differences and Influence Factors of the General Well-Being of the College Students with Left-Behind Experience
}

\author{
Zhang Yong ${ }^{1,}$, Cheng Teng ${ }^{1,2}$, Zhang Bei ${ }^{1,2}$, Tang Pei ${ }^{1,2}$, Cao Yang ${ }^{1,2,3}$, Zhou Danhua ${ }^{1}$ \\ ${ }^{1}$ School of Architecture and Urban Planning, Hunan University of Science and Technology, Xiangtan, China \\ ${ }^{2}$ School of Education, Hunan University of Science and Technology, Xiangtan, China \\ ${ }^{3}$ Mingde Dongjing Mmiddle School, Changsha, China \\ Email address: \\ 292278@qq.com (Zhang Yong)
}

\section{To cite this article:}

Zhang Yong, Cheng Teng, Zhang Bei, Tang Pei, Cao Yang, Zhou Danhua. Study on the Differences and Influence Factors of the General Well-Being of the College Students with Left-Behind Experience. Education Journal. Vol. 4, No. 5, 2015, pp. $283-289$.

doi: $10.11648 /$ j.edu.20150405.24

\begin{abstract}
The problem of left behind children has been a concern of China's social and economic development for a long time. As a relatively special group, some of the first generation of left behind children has entered the university, and they are an important part of the backbone of the country in the future. Therefore, it is important to pay attention to their healthy growth. By the methods of a questionnaire survey, taking Hunan University of Science and Technology students as research objects, this paper attempts to analyze the general well-being characteristics and its influencing factors. The results show that: (1) the general well-being of college students is correlated with the left-behind experience. The general well-being of the college students with left-behind experience is lower than the national norms and the college students who have not the left-behind experience. Besides, the latter is greater than the former; (2) the general well-being of college students with left-behind experience is correlated with differences in the time, sexuality, grade and specialty, and so on. The total well-being score of the college students with left-behind experience is greater than that of the college students who have spent a few times on left-behind. And for the jolly factors, the score of the schoolboy is higher than the schoolgirl. Meanwhile, the total well-being score of the senior students is lower than that of the junior students. The general well-being of the liberal art students is lower than that of the science students. On these bases, the paper provides some suggestions and countermeasures.
\end{abstract}

Keywords: General Well-Being, Left-Behind Experience, College Students

\section{Introduction}

Sense of well-being is an important psychology parameter which reflects the quality of individual life in the sociality. It refers to a kind of mood state while the individual needs are met, and their ideals are realized [1]. The general well-being is an important signal which is used to measure a group quality of life with same background. And it is also an important research field of positive psychology [2, 3, 4]. General well-being schedule, made by the United States National Center for health statistics, is a check tool which is used to evaluate those tested on happiness statement. In fact, many scholars in China have already carried out a series of research on the college students' General well-being. Zhang Lian, etc. studied the general well-being of students in independent college through investigation and found that these students' General well-being was related to their gender, locally-granted student and major [5]. Taking students of Minzu University of China as the research object, the research results by Zhou Yijun et al. showed that sex, nationality, family income, etc. had no effect on the General well-being, however, learning achievement, stature appearance, morality level, social relations had significant effects on the General well-being [6]. From the angle of the college graduates in local colleges, Tang Lei studied the relationship between the employment, school work, love situation, temperament style, etc. and the general well-being [7]. He Shude explored the relationship between college students' psychological capital and general well-being [bbbb]. At the same time, He Shude also studied the general well-being issues of college students $f$ rom the most serious 
disaster-stricken areas [cccc]. Li Junling carried out the correlative study on the relationship between college students' attachment types and general well-being [dddd]. The problems for children with left-behind experience have long been an issue of concern during the process of China's social and economic development. A series of social problems caused by the left-behind children had already become the hotspot of research. However, the present research has mainly focused on underage left-behind children, the research on the first generation of left-behind children who were born in the late 1980s and the early 1990s and already being the university youth is less. And this part of students in universities already occupied a large proportion; they are the future builders of the country, but also a special group. In fact, previous studies have shown that college students with left-behind experience have the characteristics of high depression levels, low self-evaluation, emotional instability, interpersonal withdrawal, the psychological development imbalance, etc [11-15].

In fact, the college students with left-behind experience have grown up under the relative lack of parental affection status and family environment. So, their psychological development has already changed greatly. Exceptional growth environments make them easier to perceive the external pressure and experience a variety of confusion and emotional changes. Therefore, the investigations on general well-being of the college students with left-behind experience are more helpful for people to understand their adult psychological development status. Based on each factor score difference of their general well-being, people can also find some problems about this special community to probe into the reasons for the difference. Obviously, according to the problems, it is of major advantage to help their healthy growth through given proper advice and opinions.

Therefore, taking the students in Hunan University of Science and Technology as research subjects, this paper analyzed the general well-being by a questionnaire survey method, and the research results will supply the reliable reference for Chinese left-behind children problem research.

\section{Materials and Methods}

\subsection{Investigation Subjects}

300 students (147 boys, 153 girls) in Hunan University of Science and Technology are selected based with hierarchical sampling method randomly as a research object. 274 valid questionnaires were obtained and the effective rate was $91.33 \%$. There are 132 college students with left-behind experience, including 61 boys and 71 girls; and there are 142 college students without left-behind experience, including 66 boys and 76 girls. All respondents are from different grades and different majors.

\subsection{Research Methods}

General well-being scale consists of 33 options, among the options, 1, 3, 6, 7, 9, 11, 13, 15, 16 are the backward score.
And each question has a corresponding fraction options. The total score is the sum of the front 18 options. And the higher total score gets, the higher the happiness is. In addition to assessing general well-being, this scale can also be used to assess 6 factors of happiness through its 6 subscales according to different contents. These 6 factors include the health concern factor, energy factor, life satisfaction and the interest factor, doldrums or pleasant mood factor, the control of emotion and behavior factors and relaxation and tension (anxiety) factor. The coefficient between a single-item score and the total score of the scale ranges from 0.48 to 0.78 . The coefficient between the subscales and total scale ranges from 0.56 to 0.88 . The coefficient of internal consistency is 0.91 in male and 0.95 in female. After an interview with experts, the total score of GWB on depression assessment of consistency is 0.27-0.47. And compared with Anxiety Scale (PEI, PSS and CHQ), the consistency is $0.41,0.40$ and 0.10. And compared with Depression Scale (HQ, Zung and MMPI), the consistency is $0.35,0.28$ and 0.21 . And compared with PFI depression scale, the consistency is higher (0.50) [5]. Based on GWB, the investigation data are integrated and analyzed according to the differences of left-behind experience or not, gender, major, grade and left-behind time.

\section{Analysis and Results}

\subsection{General Well-Being Scores of College Students with Left-Behind Experience are Lower than that of the National Norm}

It showed that the general well-being scores of undergraduate male and female with left-behind experience were all below the national norm (Table 1). Especially, the general well-being scores of the boys with left-behind experience are significantly lower than the national norm. In fact, the college students with left-behind experience in their childhood often come from rural areas and their family economic situation is not good. So, special growth environments make them more introverted and poor communication. Thus, they don't like to participate in campus cultural activities in the university and their lives are relatively simple and monotonous. And they do not make good use of the opportunity of contact with the outside world to express themselves and have fewer friends than the other college students. It can be seen that all these reasons above are the main causes of their low happiness. In addition, high-contrast daily consumption can be effectively released and born for the college students with better psychological quality. But under the double pressure of material and spiritual, some left-behind students could not objectively view the difficulties in life to produce a strong sense of inferiority. So, they always worry that others feel ashamed of them, isolate themselves and are not good at showing themselves in public.

Due to inferiority and pessimism, their ideologies have long been in a passive state, so they look at matters with pessimistic and bias mood. And they often complain of 
injustice. Also because of the autistic and the lack of communication, etc., their world view is not broad enough [11-15].

Table 1. Comparison of total GWB score of college students with left-behind experience and national norm.

\begin{tabular}{lll}
\hline & College students with left-behind experience & National norm \\
\hline Boys & 71.377 & 74 \\
Girls & 70.943 & 71 \\
\hline
\end{tabular}

\subsection{Gender Differences of General Well-Being and Factors Among College Students with Left-Behind Experience}

Table 2 showed that there was significant difference of the factors' scores in health and worry, depression and happiness and emotional behavioral control between college boys and girls with left-behind experience. It was evident that boys scored lower than girls in health-worry factor and emotional behavioral control. And in depression-happiness factor, boys scored lower than girls significantly. However, in other factors, the score difference of boys and girls is less obvious. Actually, the reasons which cause the factor differences of the gender lie in social and cultural. The formation of gender consciousness is a long socialization process and the values are also products of personal socialization. So, the individual consciousness is inevitably biased by the sex characteristics. But for women, they pay more attention to beauty, compassion, life and wish to be recognized.

Table 2. Gender differences of General well-being and factors among college students with left-behind experience.

\begin{tabular}{lll}
\hline & Boys & Girls \\
\hline General well-being & 71.38 & 70.94 \\
Health and worry & 7.28 & 7.83 \\
Depression and happiness & 14.38 & 14.49 \\
Emotion behavioral control & 10.17 & 10.27 \\
Energy & 17.93 & 17.24 \\
Satisfying the interest & 6.30 & 6.23 \\
Relaxation or stress & 15.00 & 14.90 \\
\hline
\end{tabular}

\subsection{Comparison of General Well-Being and Factors of College Students with Different Left-Behind Duration Time}

The left-behind duration time will affect the general well-being of college students. The data showed that the longer the college students had the left-behind experience, the lower their happiness was. The general well-being of the college students with left-behind experience time in one year has even exceeded the national norm and the factors' scores are relatively high. Factors' scores of satisfying the interest and general well-being of the college students with three years left-behind experience were significantly lower than those of the college students with shorter than two-year left-behind experience. And scores of depression and happiness, emotional behavioral control and energy are also lower among the college students with three-year left-behind experience (Figure 1). The study found that the longer the parents went out to work, the more serious the situation which the left-behind children and parents were separated into two different worlds by the time and space distance was. As a result, the contact and communication frequency between parents and left-behind children is also reduced. During the critical period of child growth and transition from babyish to mature trend, the role of parents is very important. The longer the role of parents is lacking, the more deeply the children's growth has been affected.

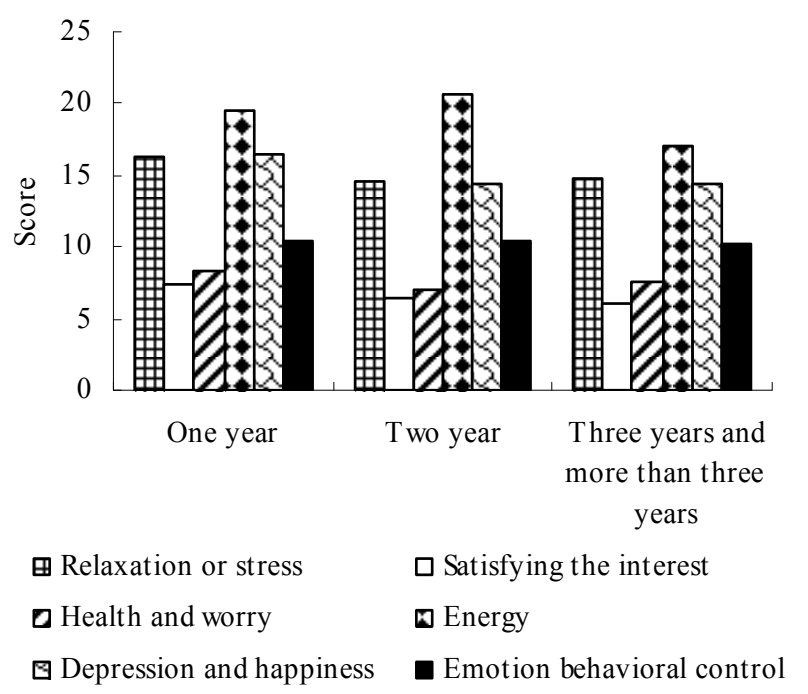

Figure 1. A Comparison of General well-being and factors of college students with different left-behind duration time.

\subsection{Comparison of the General Well-Being and Each Factor in Each Grade Among the Students with Left-Behind Experience}

The growth process of college students is a mature and independent process gradually, so there may be differences of the general well-being among different ages and different grades for the students with left-behind experience. Table 3 shows the general well-being of students with left behind experience and each factor of the different grade. It was clear at first glance that the general well-being of the sophomores with left-behind experience is significantly higher than that of the other grade students. Moreover, the main difference is the anxiety factor and the differences of the other factors are less evident. In addition, it also can be observed that the score of the anxiety factor of the senior students was the lowest, obviously, such people have the highest tension degree in their lives. At the same time, the energy factor of these people has also a higher score (Table 3 ).

Table 3. Comparison of the General well-being and each factor in each grade among the students with left behind experience.

\begin{tabular}{llll}
\hline Item & Freshman & Sophomore & $\begin{array}{l}\text { Junior and } \\
\text { senior }\end{array}$ \\
\hline General well-being & 69.78 & 72.64 & 69.63 \\
Health concerns & 7.45 & 7.83 & 7.05 \\
Energy & 17.24 & 17.66 & 18.05 \\
Meet the interest & 6.22 & 6.27 & 6.32 \\
Sad and happy & 14.29 & 14.32 & 14.32 \\
Emotional behavior control & 10.08 & 10.38 & 10.05 \\
Relaxation or tension & 14.49 & 15.63 & 13.84 \\
\hline
\end{tabular}




\subsection{Comparison of the General Well-Being and Each Factor in Each Grade Among the Arts and Science College Students with Left-Behind Experience}

The college teaching system which is separated into science teaching and art teaching can impact on college students' characters and their future occupational development. As a result, this education system makes them have large or small differences in enterprise, sense of achievement, domination and good health, etc [16, 17]. According to Table 4, it can clearly be seen that the general well-being and the scores of different factors of the students (the art and science major) with left-behind experience in Hunan University of Science and Technology existed differences certainly. Analysis shows that energy and depression factors have a significant difference between every factor. However, although other factors are different, but not too obvious.

Table 4. Comparison of the General well-being and each factor in each grade among the arts and science college students with left behind experience.

\begin{tabular}{lll}
\hline Item & Arts students & Science students \\
\hline General well-being & 70.91 & 71.31 \\
Health concerns & 7.51 & 7.62 \\
Energy & 17.82 & 17.38 \\
Meet the interest & 6.33 & 6.21 \\
Sad and happy & 14.25 & 14.82 \\
Emotional behavior control & 10.11 & 10.30 \\
Relaxation or tension & 14.89 & 14.99 \\
\hline
\end{tabular}

Note: arts majors include Chinese, history, foreign languages, political research, primary education and secretary etc. Science majors include mathematics, life science, biotechnology, computer, chemistry, geography, etc.

\subsection{Difference of Each General Well-Being Factor Between Students with Left-Behind Experience and Students Without Left-Behind Experience}

Figure 2 shows that the general well-being values of students with left-behind experience were significantly lower than that of students without left-behind experience clearly. Moreover, there is significant difference among anxious factor, sad and happy factor and energy factor. However, the difference of the other factors becomes less obvious. As everyone knows, most students with left-behind experience come from the township. Along with children's growth, the time to spend with their parents will continue to reduce and the importance of peer relationships in children's socialization process is strengthening increasingly, but for the vast majority of children, they will still have strong dependence on their family. For adolescents, during this process, they maintain good communication with their parents in all aspects, and parent still is the principal source of acquiring advice and emotional support. But as students with left-behind experience, they often worry about parental health, family economic situation and their own sense of security. So they didn't like their parents frequently to travel abroad. Moreover, with the age increasing, the desire for family integrity will be stronger. Obviously, there is little doubt that the emotional experience in childhood will affect the future personality development. As the results, left-behind children always show the self-enclosed character. The reason is that some of these children have a serious sense of loss while both parents go out. During a period of time, these children don't like to talk and communicate with others and are depressive personality. Peer group is an earliest group which children must be faced in their early children's socialization. When the left-behind children wronged in peer relationship, children often feel helpless because they don't get the care of their parents and bad mood also did not have the proper vent. In fact, communication problems always will lead to further psychological problems. Introverted children are not hard to form a sense of inferiority and solitary.

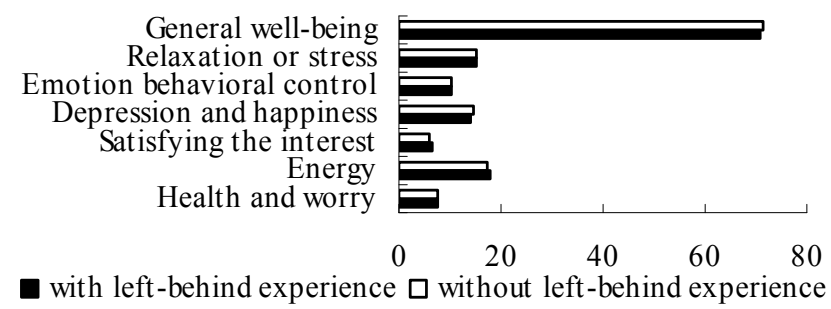

Figure 2. Comparison of General well-being and factors of college students with different left-behind duration time.

\section{Analysis of Influencing Factors}

The general well-being of college students in Hunan University of Science and Technology in this investigation is higher than that of the previous research results. The National General well-being norm for boys is 74 and 71 for girls. However, according to this investigation and analysis, the general well-being is higher than the national norm, especially the girls. The general well-being of college students in Hunan University of Science and Technology in this investigation is 74.43 for boys and 73.55 for girls. The survey showed the general well-being of the college students with left-behind experience scored lower than the national norm, especially the boys with left-behind experience.

\subsection{Relationship Between Sexuality and General Well-Being}

From the average general well-being, boys scored 71.38 and are slightly higher than that of the girls' 70.94. However, the difference is not so noticeable. The boys' scores in the energy, life satisfaction and interest are all higher than that of the girls' slightly. This situation may be correlative with the demand that most boys live on the simple life. For example, it can be seen that the girls are always thoughtful, more delicate and sensitive and emotional fluctuations, especially for their health and feeling deeply to the environment changes. For the factors of depression and happiness and emotional behavioral control, the girls scored higher than the boys, and this result is consistent with other researcher results 
[18]. For the factors of health and worry, the boys scored lower than the girls. However, for the factors of energy, girls scored higher than boys. This result showed that the boys are more worried and concerned about their health. The boys on health concerns may be related to Chinese traditional culture of the different sexuality role in the society. Actually, males are generally expected as the "brave, strong, dominant, leader" part. On the contrary, females are always expected to the "considerate, submissive" part [19]. The stereotype concept of the sexual role makes the males to take more family and social responsibility. So, males must pay more attention to their body, because the males need to have a strong body to bear those responsibilities. In addition, the male is usually also expected to "strong, powerful", and the female was expected to "weak". So, the male intended to comply with a society sexual role to pay more attention to their health, this may be the other reason. At the same time, although Chinese "labor laws" expressly provides that females shall have equal rights as males in employment, the inequalities between the sexes in employment market are still serious. Some employers even declared that they only enrolled boys. Especially, this sex discrimination phenomenon plays an important role in science and engineering specialty. Due to getting into trouble on all sides and frustrated, part of the female college students gradually lost the courage to apply for a job, or anger and resentment to the society [20].

\subsection{Correlations Among the Length of Left-Behind Time and General Well-Being and Each Factor}

The length of left-behind in childhood can affect their energy factor, the life satisfaction and the general well-being when they reach adulthood. The survey results in Hunan University of Science and Technology show that the index of the general well-being was reduced with the extension of the left-behind time. Actually, parents play a key role in the growing of children. Among the family factors, parental rearing patterns are one of the most important factors which affects children's social development and mental health [21] and are also an important early influential factor of the development of individual self-esteem. And it always continues to perform a role in their lives [22]. The child's character and habits will indirectly be affected by the family education patterns, so, if parents are away from their children and left the children alone in the critical growth period of the children's mental development, the children's inward sense of security, the family sense of dependence and the sense of responsibility will be weakened under the neglected emotional growth environment. So the longer students left behind, the fewer students are educated by the good family education. In fact, under such a growth environment, the students are more likely to accept some inappropriate educational mode from family, such as indulged and spoiled, abuse with violence speech, over protection and giving the child less emotional warmth, etc.

\subsection{General Well-Being's Difference of Students with Left-Behind Experience in Different Grades}

This survey shows that the general well-being of the students also appear obvious differences among different grades. The highest score was the sophomore at this university. Scores of senior students' relaxation or tension factor were the lowest, and it can be seen that the anxiety degree was the highest. On the contrary, the anxiety degree of the freshmen is the lowest. The reason is that the freshmen were released from the high school life and their moods are particularly relaxed. In this period, they are positive, optimistic, curious to face everything. Moreover, the academic burden is lighter, and it is not affected by the pressure of employment. However, in recent years, the increasing pressure of employment makes the mental burden of senior students, and then leads to the detection rate of anxiety disorders increasing significantly [23]. Some problems, such as poor control ability, emotional problems and behavior problems, are easy to appear. So the general well-being is low [24].

\subsection{Differences of the General Well-Being Between Arts and Science Students}

The survey found that a major study had significant effects on the general well-being of college students. The research shows that the index of the general well-being of liberal arts students is lower than that of the science students. Except the factor of sad and happy of the arts students with left-behind experience lower than that of the science students significantly, the differences of the other factors are not particularly significant. In fact, the way of thinking is different between the liberal arts students and science students. Generally, liberal arts students are good at imaginative thinking (emotional thinking) and science students are good at the abstract thought (logical thinking). Relative to science students, arts students are more sentimental, emotional instability and pay more attention to their own inner world, self-enclosed, sensitive, suspicious, stubborn and not strong independence because of learning more knowledge about history, politics and literature in their study life. Consequently, liberal arts students more susceptible and sensitive to the surrounding environment changes so that the degree of depression is higher than the science students. As we know, liberal arts and science are homologous, and all of them are the knowledge categories in the process of human development. It is difficult to sum up the characters only based on the texts of "liberal arts" and "science". So, the difference of general well-being between "liberal arts" and "science" is not particular.

\subsection{Difference of General Well-Being and its Different Factors Between Students with Left-Behind Experience and Students Without Left-Behind Experience}

The survey shows that general well-being of students with left-behind experience is significantly lower than that of other students. The differences were primarily expressed 
in the differences of anxiety factors and depression or happy factors, but the difference is not significant in other factors and general well-being. This may be the consequences of family conditions and the impact of later education. Through face-to-face interviews, most college students with left-behind experience had come from undeveloped towns or remote villages. As a result, for college students with left-behind experience, they may face greater economic pressure and insecurity, which may be the cause of higher scores on anxiety factors.

\section{Conclusions and Countermeasures}

The general well-being of the college students with left-behind experience can be summed up as the following: the general well-being of the college students with left-behind experience is lower than the national norm and other students within the school; the longer the left-behind time and the higher the grade, the lower the overall well-being; For the college students with left-behind experience, the general well-being of the liberal art students is lower than that of the science students; the female students' general well-being is lower than that of the male students.

\subsection{Create a Good Atmosphere, Enhance Students' Interaction and Strengthen the Exchange of Teachers and Students}

It should strengthen the culture construction of the class and dormitory. Through the mutual love, mutual care and love between teachers and students and among students, it can set up a warm, harmonious unity in the collective atmosphere. In order to resolve the adverse effects caused by loneliness, other negative emotions and economic pressure, it should strengthen communication with the ineloquent and introversion students, especially with the college students with left-behind experience to let them feel more collective warmth.

In addition, it should give full play to the advantage of school groups, the role of students union and social organization and carry out psychological mutual aid activities actively. The purpose which most of the parents in rural areas are out of work is to hope that their next generation can jump out of their poverty and backwardness living environment and make a living by knowledge rather than a labor-man. So for these families, cultivating a college student is hard. Therefore, in order to make the "fortune's favored children" healthy growth, colleges should pay attention to the psychological education of this students group, keep abreast of their psychological dynamics, intensify ideological education efforts. Especially, through strengthening the new-school education and developing their proper values to make him confront the distance between ideals and realities.

\subsection{Adjust your Attitude to Face Increasingly Fierce Employment Pressure with Nonchalant Ease}

Having expectations for the future is a psychological orientation. All college students who just entered the community became immersed in pursuing the ideal job to realize their own value. However, for college students with left-behind experience, their poor family economic situation makes them learn knowledge with a mentality of "Carps jumping over the Dragon Gate". At the same time, the family had to invest a considerable amount of capital for their children to go to college, even debts for their children to study. Under such realistic pressure, these students are eager to get a return in the short term after completing their studies. However, the employment situation is more severe, the results are rarely satisfactory. Especially for college students with left-behind experience, the high degree of anxiety and potential pressure has let them bear the inferiority and tension of their peers. So, once they encounter difficulties and setbacks, due to the lack of the necessary ideological preparation, it often leads to a low evaluation of their own and the loss of confidence in the future. A sharp shock brought about by the contrast between reality, and dream will have some students lost their confidence in their knowledge, ability and even the future. Faced with these problems, it is no use to complain, but it is important to adjust the mentality attitude of self, and improves their psychological ability to all kinds of unexpected events. In fact, the process of employment is also the process which the students to recognize themselves, understand society, and actively adjust themselves to adapt to the society. Stay calm and maintain clear thinking to analyze the cause of their failure when they encounter setbacks during the process of job hunting. At the same time, they should regard the process of job hunting as a good opportunity to understand society, understand professional lives and adapt to society. They should develop themselves through the job hunting and promote self-maturity [25].

\section{Acknowledgements}

Financial support from teaching reform project of "The research and practice of cooperative education mode of geographical science" (supported by Hunan Provincial Education Department); Project of Science and Technology Department of Hunan Province (2013RS4053); Social Science Fund of Hunan Province (12YBA136).

\section{References}

[1] Ed Diener, Eunkook M. Suh, Richard E. Lucas, and Heidi L. Smith. "Subjective well-being: three decades of progress." Psychological Bulletin, Vol. 125, 1999, pp. 276-302.

[2] Fazio AF. "A concurrent validation study of the NCHI general well-being schedule." Hyattsville MD. National Center for Health Statisticsm, 1997.

[3] Diener E. "Subjective well-being." Psychological Bulletin, Vol. 95, 1984, pp. 542-575.

[4] MA Rui-jie, LIU Qian-qian. "General Well-being Status of Undergraduates in Kunming Medical University.” Journal of Kunming Medical University, Vol. 36, 2015, pp. 174-176.aaaa 
[5] Zhang Lian, Wang Kun. "A survey of general well-being of students in independent institute." Sichuan Mental Health, Vol. 23,2010 , pp. $220-222$.

[6] Zhou Yijun, Tian Jin, Zhou Lijing, Yun Miaoying, Zhang Sha. "Study on general well-being of undergraduates in Minzu University of China." Journal of the Central University for Nationalities, Natural Sciences Edition, Vol. 18, 2009, pp. 81-85, 89 .

[7] Tang Lei. "A study on the relationship between the main life events, temperament type and general well-being of the undergraduates in local college." Journal of HuBei TV University, Vol. 29, 2009, pp. 50-51.

[8] He Shude. "On the Relationship between College Student' General Well-being and Psychological Capital." Sichuan University of Arts and Science Journal, Vol. 25, 2015, pp. 85-87. bbbb

[9] He Shude. "The General Wel 1-being Issues of College Students f rom the Most Serious Disaster-stricken Areas. He Shude. Educational Science." China Journal of Heal th Psychology, Vol. 21, 2013, pp. 245-248. cccc

[10] Li Junling. "Correlative study on the relationship between college students' attachment types and general well-being." Journal of Chifeng University (Natural Science Edition), Vol. 25, 2015, pp. 85-87. dddd

[11] Zhang Lihua. "Psychological analysis of college students with left-behind experience." Contemporary Youth Research, Vol. 12, 2006, pp. 28-30.

[12] Yang Shumin, Li Sumin, Li Jiangxiu et al. "Survey on depression disease rates of college students with left-behind experience." Chinese Journal of Health Statistics, Vol. 25, 2008, pp. 417-418.

[13] Xu Jiancai, Deng Yuanping. "The effect of the experiences of rural home-left life on the development of college students' personality." Journal of Changchun University of Science and Technology, Social Sciences Edition, Vol. 21, 2008, pp. 123-126.

[14] Hu Jianghui, Li Qian, Zhao Wenjian, Liu Weiwen, Tang Mengjuan. "The analysis of psychological health of college students with guarded experience and ways to solve it."
Researches in Medical Education, Vol. 7, 2008, pp. 439-440,448.

[15] Wen Yiyuan, Zeng Jianguo. "Relationship between personality and mental health among college students with left-behind experience." Chinese Journal of Public Health, Vol. 26, 2010, pp. 146-147.

[16] Zhu Qingqing, Hua Jing, Gu Guixiong. "Examination of the 320 university students' Psychological health in Suzhou." China Preventive Medicine, Vol. 8, 2007, pp. 95-97.

[17] Jiang Yali, Qi Yajing. "Study on the influence of personality factors of arts and science college students on the development of the vocational development." Consume Guide, Vol. 5, 2009, pp. 237.

[18] Zhang Wen, Zheng Richang. "Subjective well-being in college students." Chinese Mental Health Journal, Vol. 18, 2004, pp. 61-62,44.

[19] Xu Dazhen. "Study on the gender stereotypes effect of gender." Psychological Science, Vol. 26, 2003, pp. 741-742.

[20] Zhang Lin, Ding Weiming. "Reflection on the inferior group among university graduates." Journal of Hunan University of Science and Engineering, Vol. 27, 2006, pp. 239-241.

[21] Gao Xueping, Yu Suwei, Su Linyan. "The relationship between hyperactive-impulsive behavior in children/adolescents and parental rearing patterns." Chinese Journal of Clinical Psychology, Vol. 11, 2003, pp. 36-38.

[22] Chen Chen. "The changing and analyzing on the study of family parenting patterns." Journal of Nanjing Normal University, Social Science, Vol. 6, 2002, pp. 95-103, 109.

[23] Zhang Siming. "Survey of the psychological anxiety of college students in a university." Modern Preventive Medicine, Vol. 35, 2008, pp. 2925-2926.

[24] Liu Rengang, Gong Yaoxian. "Subjective well-being and stress level in elderly." Chinese Mental Health Journal, Vol. 15, 2001, pp. 28-30.

[25] Liu Jian, Liu Rixian, Zhu Hongtao. "College students' psychological characteristics and their self-adjustment relating to employment." Journal of Shanxi College for Youth Administrators, Vol. 19, 2006, pp. 32-33. 